\title{
On-Line Monitoring of Photometric, Colorimetric, Electrical and Thermal Parameters during LED Accelerated Life Testing
}

\author{
Lin Zhou ${ }^{1}$, Yimin $\mathrm{Hu}^{1}$, Wenpeng Xiao ${ }^{1}$, Minggao $\mathrm{Cao}^{1}$, Hailin $\mathrm{Wu}^{2}$, Bing Zhang ${ }^{3}$, Gang Jing ${ }^{1}$ and Yan Liu ${ }^{1, *}$ \\ ${ }^{1}$ Optomechatronics laboratory, Research Institute of Tsinghua University at Shenzhen, P.R. China \\ ${ }^{2}$ College of materials engineering, Shenzhen University, P.R.China \\ ${ }^{3}$ College of Mechatronics and Control Engineering, Shenzhen University, P.R.China \\ *Corresponding author
}

\begin{abstract}
In practice, one can get luminous flux parameter of LED by off-line measurement method and make prediction of the LED module's service life time. But the prediction results only focus on LED luminous flux, meanwhile colorimetric and thermal characteristics are not involved. Towards to the disadvantage of present LED life time test which only pay attention to the decay of emitting device's luminous flux and efficacy, this paper introduces a kind of innovative LED accelerated life test system and method. During the test, the system can load temperature, humidity, vibration, current and other stress to LED modules in the same time, meanwhile the system can also measure photometric, colorimetric, electrical and thermal parameters of LED modules at the real time of accelerated life test. One can make pre-judgment to the reliability and failure mode of LED module according the variation trend of photometric, colorimetric, electrical and thermal parameters.
\end{abstract}

Keywords-photometric-colorimetric-electrical-thermal on-line measurement; accelerated life test; led

\section{INTRODUCTION}

The lighting industry has already reached a consensus that due to its long life, high efficiency and other advantages, high power white LED will replace the traditional lighting source gradually. However the practical service life time is far less than 100 '000 hours as ideal expectation cause of obvious reliability ${ }^{[1]}$ problems.

Aiming at the prediction of LED's reliability, domestic and foreign researchers haven't get a common view yet. In practice, one can get luminous flux parameter of LED by off-line measurement method and make prediction of the LED module's service life time according the IES LM-80-08 and IES TM-21-11 standard principle ${ }^{[2-3]}$. But the prediction results only focus on LED luminous flux, meanwhile colorimetric and thermal characteristics are not involved.

As is known to all, for LED lighting applications, except for the photometric parameters such as luminous flux and luminous efficiency, color temperature and color rendering index etc. colorimetric parameters are also very important to quality of illumination, meanwhile the LED junction temperature, thermal resistance and related thermal parameters carry critical meaning to its service life and luminous efficiency. Therefore, study the changing trend of photometric, colorimetric and thermal parameters at same time in process of LED aging test has vital significance.

\section{EQUIPMENT SYSTEM DESIGN}

The entire testing system configuration is shown as Figure 1. The main components include: high-low temperature-humidity test box, vibration generator, LED multi-channel DC power supply, LED module holder, LED thermal properties measurement system, light collection system (integrating sphere and its driving motor), spectrometer and computer.

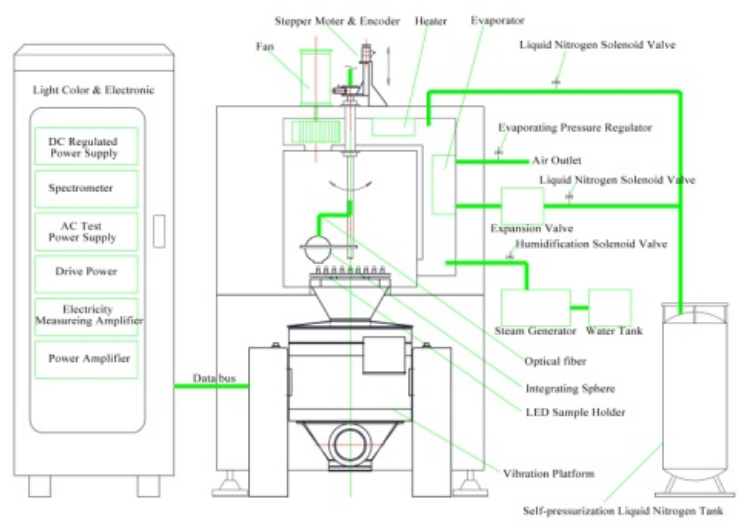

FIGURE I. SCHEMATIC DIAGRAM OF LED MULTI STRESS ACCELERATED LIFE ON-LINE TESTING SYSTEM

During the test, temperature-humidity test box, vibration generator, LED power supply, light collection system and spectrometer are all controlled and coordinated by the PC host. PC host automatic control the main components to load temperature, humidity, vibration and current and other stress according the program which was set previously to LED modules involved in the accelerated life testing. The integrating sphere will collect most of light emit by LED module and import it into spectrometer, complete on-line measurement of photometric, colorimetric and electricity parameters for each sample. Meanwhile, LED thermal parameter measurement system will complete on-line measurement of junction temperature and thermal resistance for all the samples. 
According to multi-parameter changing trend of the test samples, the expected performance of the test sample is predicted by the relevant algorithm. Prototype of the system is shown as Figure 2.

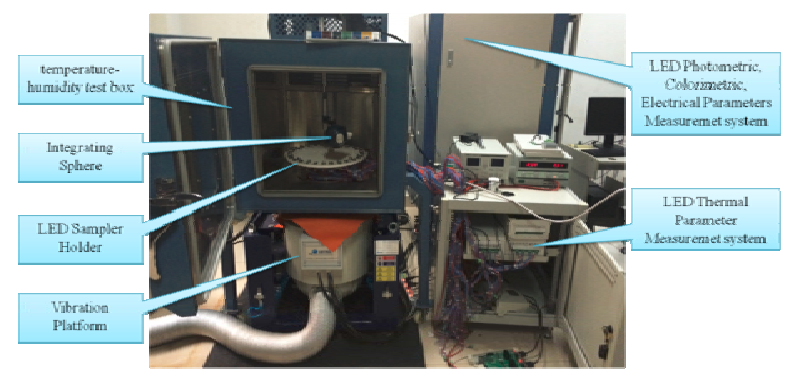

FIGURE II. PROTOTYPE OF LED MULTI STRESS ACCELERATED LIFE ON-LINE TESTING SYSTEM

\section{ON-LINE MEASUREMENT OF PHOTOMETRIC, \\ COLORIMETRIC AND ELECTRICITY PARAMETERS OF LED}

The photometric, colorimetric and electricity parameters of LED mainly include: relative spectral power distribution, flux and radiation flux, chromaticity coordinates, dominant wavelength, peak wavelength, correlated color temperature, color index, forward voltage, current, power, luminous efficiency and etc.

The photometric, colorimetric and electrical parameters of LED are measured by integrating sphere and spectrometer. Integrating sphere is installed inside the testing box as shown in Figure 3 and will driven to move vertically and counter-clockwise by the motor which was placed above it. The integrating sphere will collect most of light emit by LED module and import it into spectrometer, complete on-line measurement of photometric, colorimetric and electrical parameters for each sample.

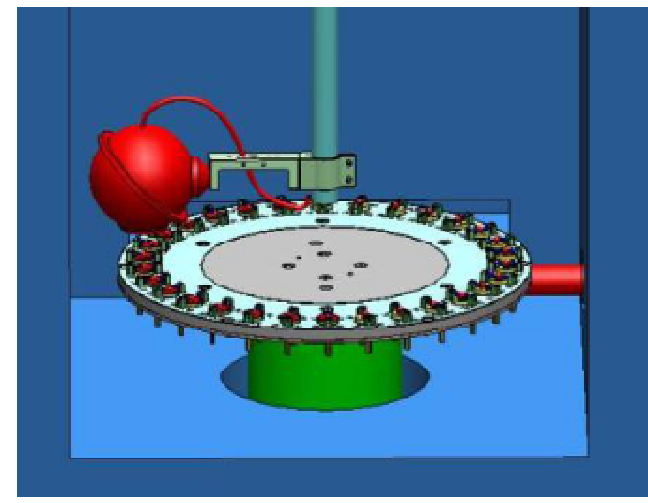

FIGURE III. SCHEMATIC DIAGRAM OF LIGHT COLLECTION SYSTEM

\section{ON-Line MEASUREMENT OF THERMAL PARAMETERS OF LED}

Thermal parameters of LED mainly refer to junction temperature and thermal resistance. Junction temperature means the temperature at PN junction zone when current flows. Junction temperature is key thermal parameter to characterize LED's performance and decide its service life and luminous efficacy directly. It has a function relationship with luminous flux, color rendering index, color temperature, peak wavelength and etc[4]. The thermal resistance of LED directly affects the junction temperature when samples are impacted by the same working conditions. In practice, the forward voltage method published in JESD51[5] is most widely used to measure LED junction temperature $\mathrm{Tj}$. Because there is a linearity relationship between forward voltage $\mathrm{Vf}$ and $\mathrm{Tj}$ under the specific test current I, as long as the Vf value measured are more than two temperature values, the relationship can be obtained.

According to electric heating analogy theory, the thermal characteristics of LED can be described equivalently by using $\mathrm{RC}$ circuit. Base on the forward voltage method, by using the relationship between transient response of LED devices to thermal step input and physical properties of each part of the package, with the aid of the Foster thermal model and the Cauer thermal model, thermal resistance - heat capacity of each part of device's package can be obtained by mathematical analysis.

\section{DISCUSSION}

Pick a group of high power LED module samples, after 48 hours activation, put them into chamber of the system. The accelerated life stresses condition were set as: (1) temperature stress is $25 \sim 1050 \mathrm{C}$, the temperature interval is $20 \mathrm{oC}$, at each temperature, the duration is 120 minutes; (2) the humidity stress is $50 \% \mathrm{RH}$; (3) the working current is $350 \mathrm{~mA}$. At every 15 minutes, the system automatically records all the LED samples' photometric, colorimetric, electrical and thermal parameters as shown in Figure 4-7.

The luminous flux - photometric parameters (Figure 4.) decreases gradually with the increase of environmental temperature, indicating that with temperature rising, photoelectric efficiency of the device became lower and lower. The color temperature - colorimetric parameters (Figure 5.) increased with the environmental temperature rising indicating that proportion of blue light in the spectrum is more and more, meanwhile the proportion of yellow light is less and less, on the other hand it could be explained as the chip's electroluminescence efficiency and yellow phosphor's photoluminescence efficiency decreased with temperature rising, but the latter's decay is stronger.

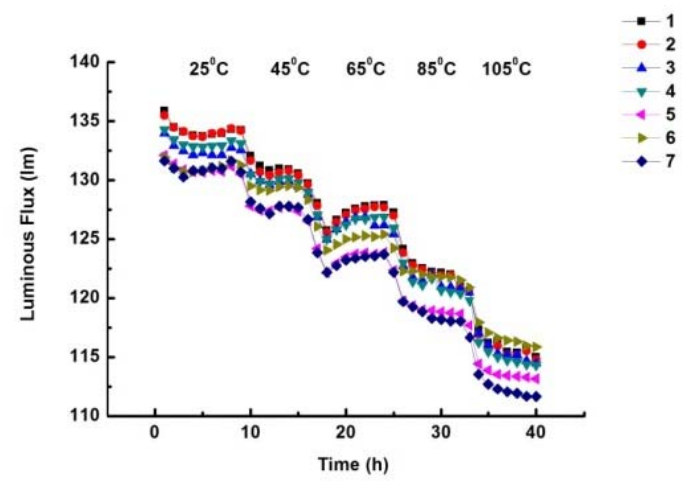

FIGURE IV. LUMINOUS FLUX CHANGING TREND WITH THE INCREASING TEMPERATURE. 


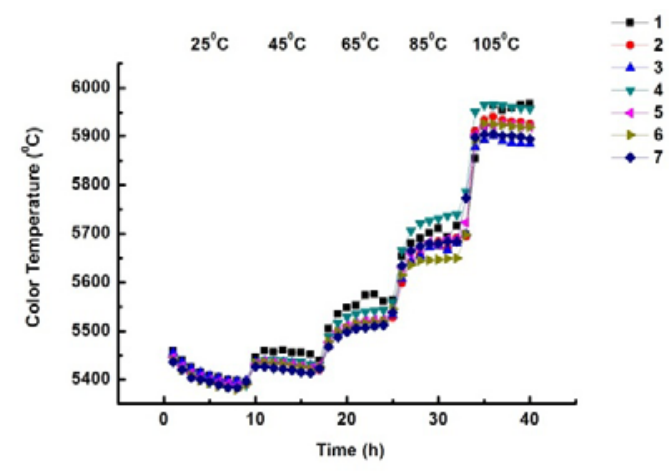

FIGURE V. COLOR TEMPERATURE CHANGING TREND WITH THE INCREASING TEMPERATURE

LED forward voltage - electrical parameters (Figure 6.) decreased gradually with the increase of environmental temperature, the intrinsic reason is that the electrical field intensity at the PN junction zone decreased with temperature rising. The junction temperature - thermal parameters (Figure 7.) has increased dramatically with the environmental temperature rising. The difference between junction temperature and environmental temperature also increased gradually. The phenomenon shown that more and more electrical power would be wasted and turned into thermal energy with environment temperature rising.

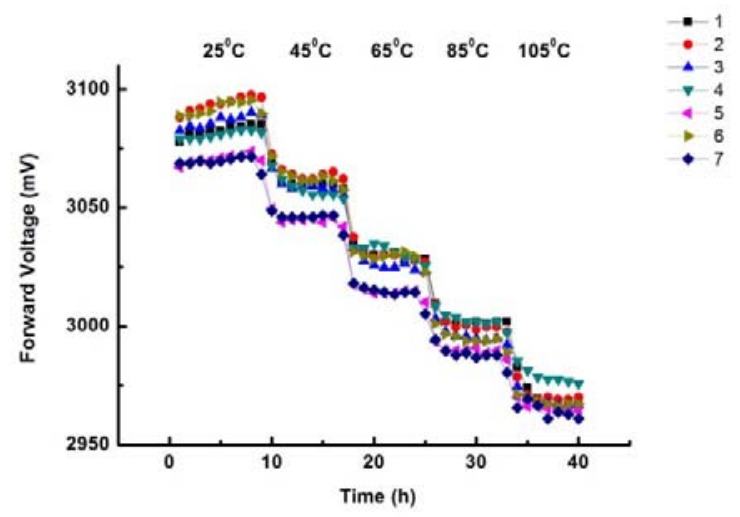

FIGURE VI. FORWARD VOLTAGE CHANGING TREND WITH THE INCREASING TEMPERATURE

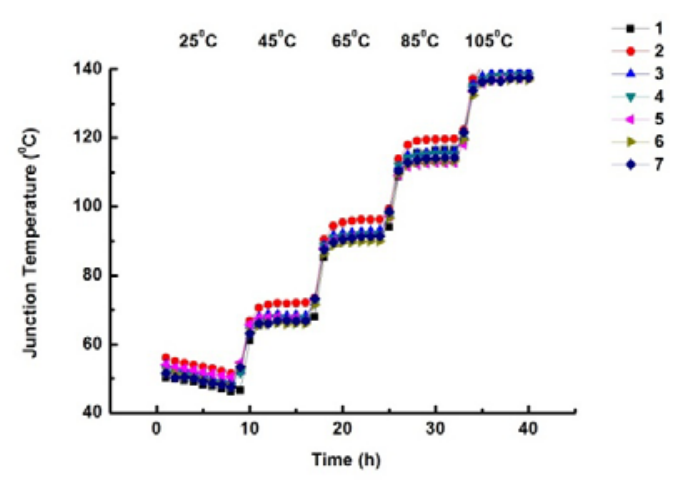

FIGURE VII. JUNCTION TEMPERATURE CHANGING TREND WITH THE INCREASING TEMPERATURE

\section{SUMMARY}

Towards to the disadvantage of present LED life time test which only pay attention to the decay of emitting device's luminous flux and efficacy, this paper introduces a kind of innovative LED accelerated life test system and method. During the test, the system can load temperature, humidity, vibration, current and other stress to LED modules in the same time, meanwhile the system can also measure photometric, colorimetric, electrical and thermal parameters of LED modules at the real time of accelerated life test. One can make pre-judgment to the reliability and failure mode of LED module according the variation trend of photometric, colorimetric, electrical and thermal parameters.

\section{ACKNOWLEDGEMENT}

This work was supported by the national key basic research and development program's (973 program, 2011CB013100) 6th Sub-topic "complex over stress accelerated life test method and reliability rules” (2011CB013106).

\section{REFERENCES}

[1] 5th Institute of Electronics fifth, Department of industry and Information Technology. Study and Analysis on the quality and reliability of LED lighting. Beijing: press of electronics industry, 2015

[2] IES LM-80-08 Approved Method for Measuring Lumen Maintenance of LED Light Sources.

[3] IES TM-21-11 Projecting Long Term Lumen Maintenance of LED Sources.

[4] EIA/JEDEC Standard JESD51-1. Integrated circuits thermal measurement method-electrical test method (Single Semiconductor Device) [S]. USA: Electronic Industries Alliance, 1995.

[5] ZHONG Wen-jiao, WEI Ai-xiang, ZHAO Yu. Dependence of GaN-based White LED Colorimetric Parameters on Junction Temperature[J]. CHINESE JOURNAL OF LUMINESCENCE, 2013, 34(9): 1203-1207. 